COMMUNICATIONS IN

ANALYSIS AND GEOMETRY

Volume 7, Number 4, 681-694, 1999

\title{
Harmonic maps and rigidity theorems for spaces of nonpositive curvature
}

\author{
JÜrgen JoST AND SHING-TUNG YAU
}

In this note, we use harmonic maps to study groups acting on products of simply connected complete manifolds of nonpositive sectional curvature with compact quotient, or sometimes more generally on simply connected complete metric spaces of nonpositive curvature in the sense of Alexandrov. By definition, that curvature condition means that if we compare a geodesic triangle in our space with a Euclidean comparison triangle of the same side lengths, then any point on one of its sides cannot have larger distance from the opposite vertex than the corresponding point in the Euclidean triangle (see $[\mathrm{N}]$ for a detailed exposition). In the case of a smooth Riemannian manifold that curvature condition is equivalent to nonpositive sectional curvature.

It was shown by Gromoll-Wolf [GW] and Lawson-Yau [LY] that if the fundamental group of a compact Riemannian manifold $M$ of nonpositive curvature has no center and splits as a product, then $M$ itself is metrically a product. We prove this result by harmonic map techniques and extend it to those more general spaces described above. In the smooth case, the required existence result for harmonic maps is due to Al'ber [A1], [A2] and EellsSampson [ES]. (In fact, Al'ber [A2] was the first to point out that harmonic maps can yield information about the fundamental group of Riemannian manifolds of nonpositive curvature.)

Gromov-Schoen [GS] then studied harmonic maps for certain nonsmooth targets. Here, however, we also have to deal with nonsmooth domains, and therefore, we need to employ the theory of the first author [J2], [J3] of generalized harmonic maps with values in metric spaces of nonpositive curvature in the sense of Alexandrov (or Busemann).

The discrete evolution method of [J2] also allows us to obtain families of harmonic maps depending continuously on some parameter. Thus, if for example the fundamental group of $M$ is a product $\Gamma=\Gamma_{1} \times \Gamma_{2}$, we let $M_{j}$ be a $K\left(\Gamma_{j}, 1\right)$ space $(j=1,2)$ and obtain two families $f_{x_{2}}^{1}\left(M_{1}\right), f_{x_{1}}^{2}\left(M_{2}\right)$, depending on $x_{2} \in M_{2}$ and $x_{1} \in M_{1}$. By the uniqueness result for harmonic maps of Al'ber [A1], [A2] and Hartman [H] as extended to the case of metric 
spaces by Gromov-Schoen [GS], these two families of harmonic maps are parallel. In case $\Gamma$ is centerfree, they will allow us to produce the desired product decomposition of $M$.

Our second theorem says that if a centerfree discrete group $\Gamma$ acts freely, properly discontinuously, and, most important of all, irreducibly on a nontrivial product Riemannian manifold $X$ with compact quotient $X / \Gamma$, and if $X / \Gamma$ is homotopically equivalent to a compact Riemannian manifold $M$ of nonpositive sectional curvature, then the universal cover of $M$ is a Riemannian homogeneous space, and there exists a totally geodesic homeomorphism between $X / \Gamma$ and $M$. If we assume in addition that $X$ is a symmetric space of noncompact type, this result is a theorem of Eberlein [E] and part of a theorem of Gromov (see [BGS]). Here, however, we do not even need to impose a curvature condition on $X$, let alone a symmetric structure. On the way of proving this result, we shall show that $\Gamma$ projects densely into the isometry group of each factor of $X$. This generalizes the Borel density theorem [B] for irreducible group actions on products of symmetric spaces. The reason why we do not obtain this result for general metric spaces is that we need a Bochner type argument in order to show that a harmonic homotopy equivalence $f: X / \Gamma \rightarrow M$ has harmonic restrictions to the local factors of $X / \Gamma$. This latter argument is the same as the one presented in [MSY]. We would like to point out, however, that this argument was already described by the second author in his address at the AMS Summer Institute on Several Complex Variables in Santa Cruz in 1989.

An exposition of the harmonic map method for studying manifolds of nonpositive curvature can be found in [J1]. Other applications include for example the investigation of finite group actions on such manifolds by SchoenYau [SY]. Another line of investigation in that area recently culminated in the harmonic map proof of geometric superrigidity by Jost-Yau [JY] and Mok-Siu-Yeung [MSY]. References for the theory of metric spaces of nonpositive curvature are [N], [J3].

The first author gratefully acknowledges the hospitality of Harvard University and generous support from the DFG, the second one support through an NSF grant.

We thank the referee for his pertinent and constructive questions.

\section{Uniqueness of harmonic maps.}

In this $\S$, we recall the uniqueness result for harmonic maps that will be the basis of our subsequent reasoning. It is due to Al'ber [A2] and Hartman [H] 
in the smooth case and was extended by Gromov-Schoen [GS] to the metric case.

Theorem 1. Let $f_{0}$ and $f_{1}$ be homotopic harmonic maps (in the sense of [J3]) between compact metric spaces $M^{\prime}$ and $M$ where the target $M$ has nonpositive curvature in the sense of Alexandrov. For $x \in M$, let $f(x, s)$ be the geodesic between $f_{0}(x)$ and $f_{1}(x)$ in the homotopy class determined by the homotopy between $f_{0}$ and $f_{1}$, parametrized proportionally to arclength by $s \in[0,1]$. Then, the length of $f(x, \cdot)$ is independent of $x$, and all these geodesics are parallel to each other. Also, all the maps $f(\cdot, s), s \in[0,1]$, are harmonic, and thus $f_{0}$ and $f_{1}$ are joined by a parallel family of harmonic maps.

Since our uniqueness statement is slightly different from the ones in the preceding references and in order to clarify the meaning of the assertions of the statement, we provide a proof based on [J3]: As explained in [J2], [J3], the energy functional the minima of which are our harmonic maps, can be obtained as the $\Gamma$-limit of functionals of the form

$$
E_{h}(f)=\int_{M^{\prime}} \int_{M^{\prime}} h(x, y) d^{2}(f(x), f(y)) \mu(d x) \mu(d y)
$$

for a given measure $\mu$ on $M^{\prime}$ and a nonnegative symmetric function $h$ : $M^{\prime} \times M^{\prime} \rightarrow \mathbb{R}$. All convexity statements employed in the sequel pertain to $\Gamma$-limits while the role of nonpositive curvature is more easily explained for the functionals $E_{h}$. Therefore, we present the reasoning for the latter.

Let $f_{0}, f_{1}: M^{\prime} \rightarrow M$ be any two maps, not necessarily harmonic. We assume that they are homotopic. Thus, there exists a map

$$
\begin{aligned}
F: M^{\prime} \times[0,1] & \rightarrow M \\
(x, t) & \mapsto F(x, t)
\end{aligned}
$$

that is continuous w.r.t. $t$ and satisfies $F(x, T)=f_{T}(x)$ for $T=0,1$. For $x \in M^{\prime}$, we let $f(x, s), s \in[0,1]$, be the geodesic arc from $f_{0}(x)$ to $f_{1}(x)$ homotopic to $F(x, s)$, and parametrized proportionally to arclength.

The essential point now is that because of our assumption of nonpositive curvature,

$$
d^{2}(f(x, s), f(y, s))
$$

is a convex function of $s$ that is even strictly convex unless the two geodesic arcs $f(x, s), f(y, s)$ are parallel in the sense that this distance is independent of $s$. In particular, in the latter case they are of the same length. (It is not 
hard to see that it suffices to consider the situation where $f(x, s)$ and $f(y, s)$ are closer than the injectivity radius of $M$.)

Since the function $h$ is assumed to be nonnegative, this implies that

$$
E_{h}[s]:=\iint h(x, y) d(f(x, s), f(y, s)) \mu(d x) \mu(d y)
$$

also is a convex function of $s$, and strictly convex unless for almost all $x, y$, the geodesics $f(x, s), f(y, s)$ are parallel. This first of all implies that $E_{h}$ is a convex function of $f$ in a given homotopy class of maps. Secondly, if $f_{0}, f_{1}$ are minimizers of $E_{h}$, then $E_{h}[s]$ is constant in $s$, and almost all geodesic $\operatorname{arcs} f(x, s), f(y, s)$ are parallel, and furthermore, $E_{h}(f(\cdot, s))$ is independent of $s$, and thus all the maps $f(\cdot, s): M^{\prime} \rightarrow M$ are minimizers as well. Also, since the geodesics $f(x, \cdot)$ are all of the same length and parametrized proportionally to arclength, the maps $f(\cdot, s)$ constitute a parallel family in the sense that the homotopy distance between $f\left(x, s_{1}\right)$ and $f\left(x, s_{2}\right)$, i.e. the length of the shortest geodesic between $f\left(x, s_{1}\right)$ and $f\left(x, s_{2}\right)$ in the homotopy class determined by the homotopy between $f_{0}$ and $f_{1}$, for $s_{1}, s_{2} \in[0,1]$, is independent of $x$. This completes the proof.

\section{Results and proofs.}

Theorem 2. Let $M$ be a compact metric space of nonpositive curvature in the sense of Alexandrov with fundamental group $\Gamma$. Suppose that $\Gamma$ has no center and splits as a product

$$
\Gamma=\Gamma_{1} \times \ldots \Gamma_{k} .
$$

Then $M$ is isometric to a product

$$
X_{1} / \Gamma_{1} \times \ldots X_{k} / \Gamma_{k}
$$

where $X_{1}, \ldots X_{k}$ are complete, simply-connected metric spaces of nonpositive curvature in the sense of Alexandrov, and $\Gamma_{j}$ acts isometrically on $X_{j}, j=$ $1, \ldots k$.

Remark 1. In order to see why the be important for our proof, it is useful to keep the $\mathbb{Z}^{2}$ action on $\mathbb{R} \times \mathbb{R}$ generated by

$$
\gamma_{1}:(x, y) \mapsto_{\mathrm{v}}(x+1, y)
$$

and

$$
\gamma_{2}:(x, y) \mapsto(x+1, y+1)
$$


in mind. Of course, this generates the same quotient as the action with generators $\gamma_{1}$ and $\gamma_{2}^{\prime}:(x, y) \mapsto(x, y+1)$. An important point in our proof will be to show that in our situation only the latter type of action can occur.

Proof. We let $M_{j}$ be a compact $K\left(\Gamma_{j}, 1\right)$ space and equip it with some metric $d(\cdot, \cdot)$ and some measure $\mu$ satisfying the conditions that are necessary to make the harmonic maps employed in the sequel continuous. In order to see that this is possible, let us briefly recall the construction of the $K\left(\Gamma_{j}, 1\right)$ space $M_{j}$ (see [W], pp. 216, 244). One starts with the Cayley graph of the group $\Gamma_{j}$ for some choice of generators of $\Gamma_{j}$. Here, the vertices are the elements of $\Gamma_{j}$, and two vertices are connected by an edge if the corresponding elements are related by multiplication with one of the chosen generators. Closed loops correspond to relations in $\Gamma_{j}$, and one caps them off by inserting two-dimensional disks. Finally, one inserts higher-dimensional cells to kill the higher homotopy groups. Since $\Gamma_{j}$ is torsion-free (because $\Gamma$ is as the fundamental group of a space of nonpositive curvature; namely, torsion elements would produce nontrivial closed geodesics homotopic to a point which cannot happen for nonpositive curvature, for example by Theorem 1 ), the resulting space is finite-dimensional, and $\Gamma_{j}$ acts freely on it to produce the desired $K\left(\Gamma_{j}, 1\right)$ space $M_{j}$ as a quotient. We may equip all the cells that are inserted with nice metrics and measures that satisfy the following properties:

(i) The construction is $\Gamma_{j}$-equivariant so that we get a metric $\mathrm{d}(\cdot, \cdot)$ and a measure $\mu$ on the quotient $M_{j}$.

(ii) The induced metrics on the lower-dimensional cells (which are contained in the boundary of more than one top-dimensional cell) agree and are nondegenerate.

(iii) The measure is the volume measure, induced by the metric on the top-dimensional cells (w.l.o.g. the metrics are Riemannian there).

(iv) The ball doubling property hulds: there exists a constant $c<\infty$ such that for all $r>0$ and all $x \in M_{j}$,

$$
\mu(\{y: d(x, y)<2 r\})<c \mu(\{y: d(x, y)<r\})
$$

(v) The Poincaré inequality holds. 
We then put

$$
h_{\epsilon}(x, y):= \begin{cases}\frac{1}{\nu(\epsilon)} & \text { if } d(x, y)<\epsilon \\ 0 & \text { otherwise }\end{cases}
$$

where $\nu(\epsilon)$ is some appropriate normalization factor, in order to make the energy functional that we are going to introduce nontrivial. We consider the approximate energy functionals $E_{h_{\epsilon}}(f)$ and their $\Gamma$-limit $E$ as $\epsilon \rightarrow 0$, as described in [J3]. By [J4], minimizers for $E$ with values in a space of nonpositive curvature are Hölder continuous.

$M_{1} \times \ldots M_{k}$ then is a compact $K(\Gamma, 1)$ space, hence homotopically equivalent to $M$, as the latter one is a $K(\Gamma, 1)$ space as well. Let $F: M_{1} \times \ldots M_{k} \rightarrow$ $M$ be a homotopy equivalence. For each $j$ and $s_{j} \in M_{1} \times \ldots \hat{M}_{j} \times \ldots M_{k}$ (the factor $M_{j}$ omitted), the method of [J2] or [J3] allows us to deform

$$
F\left(M_{j} \times\left\{s_{j}\right\}\right)
$$

into a harmonic map

$$
f_{s_{j}}: M_{j} \rightarrow M,
$$

depending continuously on $s_{j}$. (Actually, in the sequel, the $\epsilon$-harmonic maps of [J2] that can be obtained in a more elementary way would work as well as harmonic ones.) Also, by our choice of $\mu$, the maps $f_{s_{j}}$ are continuous themselves, and the map

$$
\begin{aligned}
f: M_{1} \times \ldots \times M_{h} & \rightarrow M \\
\left(s_{j}, \xi_{j}\right) & \mapsto f_{s_{j}}\left(\xi_{j}\right)
\end{aligned}
$$

is a homotopy equivalence homotopic to $F$. As such, it is in particular surjective. We then apply harmonic map uniqueness, see $\S 1$. Thus, we get $k$ families; in the $j^{t h}$ family, the leaves are of the form $f_{s_{j}}\left(M_{j}\right)$ and all isometric and parallel to each other. On the universal cover, we get corresponding families with leaves $\tilde{f}_{s_{j}}\left(\tilde{M}_{j}\right)$. (In fact, the families $f_{s_{j}}\left(M_{j}\right)$, for $s_{j}$ varying, hence also $\tilde{f}_{s_{j}}\left(\tilde{M}_{j}\right)$, yield foliations of $M$ and $\tilde{M}$, resp. (hence the terminology "leaves"), but this is not needed for our reasoning at this stage.) For $i \neq j, \Gamma_{i}$ permutes the leaves $\tilde{f}_{s_{j}}\left(\tilde{M}_{j}\right)$ (since $\Gamma_{i}$ and $\Gamma_{j}$ commute, we infer from the uniqueness statement that $\Gamma_{i}$ maps leaves to leaves), but we claim that for each $\gamma \in \Gamma_{i}, \xi_{j} \in M_{j}$,

$$
\gamma \tilde{f}_{s_{j}}\left(\xi_{j}\right)=\tilde{f}_{s_{j}^{\prime}}\left(\xi_{j}\right)
$$


for some $s_{j}^{\prime}$. We view this also in the following way: If we identify the leaves $\tilde{f}_{s_{j}}\left(M_{j}\right)$ for varying $s_{j}$ through the parallel geodesics from Thm. 1, i.e. we identify $\tilde{f}_{s_{j}^{1}}\left(\xi_{j}\right)$ with $\tilde{f}_{s_{j}^{2}}\left(\xi_{j}\right)$ for any $s_{j}^{1}, s_{j}^{2}$, we obtain a space $\tilde{f}\left(M_{j}\right)$ isometric to all the leaves $\tilde{f}_{s_{j}}\left(M_{j}\right)$ on which $\Gamma_{i}$ acts trivially. We may also identify $\tilde{f}\left(\tilde{M}_{j}\right)$ with any leaf $\tilde{f}_{s_{j}}\left(\tilde{M}_{j}\right)$, and thus consider $\tilde{f}\left(\tilde{M}_{j}\right)$ as a subset of $\tilde{M}$.

$\gamma$ then induces an isometry of $\tilde{f}\left(\tilde{M}_{j}\right)$, again denoted by $\gamma$ by an abuse of notation. If the claim is not true, there exists $x_{j} \in \tilde{f}\left(\tilde{M}_{j}\right)$ with $\gamma\left(x_{j}\right) \neq x_{j}$.

Since $\gamma\left(x_{j}\right) \neq x_{j}$ is assumed, the geodesic from $x_{j}$ to $\gamma\left(x_{j}\right)$ projects to a geodesic in $M$ different from the one defining the parallelism between the leaves coming from the uniqueness of harmonic maps. This family of geodesics yields a new homotopy between $f\left(M_{j}\right)$ and $\gamma_{*} f\left(M_{j}\right)$ to which we may again apply the uniqueness theorem for harmonic maps in order to produce a parallel family of images of harmonic maps connecting $f\left(M_{j}\right)$ and $\gamma_{*} f\left(M_{j}\right)$. We therefore obtain a nontrivial parallel family of translations of $f\left(M_{j}\right)$ inside $f\left(M_{j}\right)$, and thus also a family in $f\left(M_{j}\right)$ of parallel geodesics, denoted by $c_{x}$, going from $x$ to $\gamma_{*} x$. Let $t$ be the length of these geodesics $c_{x}$. The geodesics $c_{x}$ commute with arcs in $f\left(M_{j}\right)$ in the sense that for every $\operatorname{arc} b:[0,1] \rightarrow f\left(M_{j}\right)$ starting at $x$, we have

$$
\gamma_{*} b(1)=c_{b(1)}(t)
$$

Let $\tilde{c}_{\tilde{x}}$ be a maximal extension of a lift of $c_{x}$ to $\tilde{M}$. If it projects to a closed curve in $M$, it is not hard to see that this curve is smooth, hence a closed geodesic $c$. It then represents an element of $\pi_{1}\left(f\left(M_{j}\right), x\right)$ that commutes with all elements of $\pi_{1}\left(f\left(M_{j}\right), x\right)$; hence it is contained in the center of $\Gamma$, contradicting our assumption that $\Gamma$ is center free. If $\tilde{c}_{\tilde{x}}$ does not project to a closed curve, we take the shortest geodesic arc $b:[0,1] \rightarrow \tilde{M}$ from $\tilde{x}$ to $\gamma_{*}^{\tilde{n}} x$ for some $n$ sufficiently large so that $b$ is not contained in $\tilde{c}_{\tilde{x}}$. The family of geodesics $\tilde{c}_{b(s)}, s \in[0,1]$, then projects to a family of curves in $f\left(M_{j}\right)$. If this family defines a closed torus, we obtain a subgroup isomorphic to $\mathbb{Z}^{2}$ in the center of $\pi_{1}\left(f\left(M_{j}\right), x\right)$. Otherwise, we perform the construction with higher dimensional families until we finally obtain some closed torus and some group isomorphic to $\mathbb{Z}^{l}$ for some $l \in \mathbb{N}$ in the center of $\pi_{1}\left(f\left(M_{j}\right), x\right)$, again contradicting the assumption that $\Gamma$ is center free.

We now take another look at the assertion of the harmonic map uniqueness theorem. Namely, if we fix $x_{j} \in M_{j}$ and let $s_{j} \in M_{j}^{\prime}:=M_{1} \times \ldots \hat{M}_{j} \times$ $\ldots M_{k}$ (the factor $M_{j}$ omitted) vary, then $f\left(x_{j}, s_{j}\right):=f_{s_{j}}\left(x_{j}\right)$ is a totally 
geodesic set $\varphi_{x_{j}}\left(M_{j}^{\prime}\right):=f\left(x_{j}, M_{j}^{\prime}\right)$. In particular, the inclusion map

$$
i_{x_{j}}: \varphi_{x_{j}}\left(M_{j}^{\prime}\right) \rightarrow M
$$

is harmonic. If we now keep $s_{j}$ fixed and let $x_{j}$ vary, then by the same argument, $f\left(x_{j}, s_{j}\right)=\varphi_{x_{j}}\left(s_{j}\right)$ yields a totally geodesic set $\varphi_{s_{j}}^{\prime}\left(M_{j}\right)$ coinciding with $f_{s_{j}}\left(M_{j}\right)$, again applying the harmonic map uniqueness theorem.

By our preceding arguments, we then get two $\Gamma$-invariant totally geodesic foliations on the universal cover $\tilde{M}$, denoted by a ${ }^{-}$on top. (The foliation property easily follows from our construction and the fact that $f$ as a homotopy equivalence is surjective.) In order to conclude the proof, we shall show that they induce a $\Gamma$-invariant product structure for the metric of $\tilde{M}$. For $\gamma_{j} \in \Gamma_{j}^{\prime}=\Gamma_{1} \times \ldots \times \hat{\Gamma}_{j} \times \ldots \times \Gamma_{k}, \gamma_{j} \tilde{f}\left(x_{j}, s_{j}\right)$ is the point on $\gamma_{j} \tilde{f}_{s_{j}}\left(M_{j}\right)$ closest to $\tilde{f}\left(x_{j}, s_{j}\right)$. Namely, otherwise the geodesics on the leaves $\gamma_{j} \tilde{f}_{s_{j}}\left(M_{j}\right)$ between that closest point and $\gamma_{j} \tilde{f}\left(x_{j}, s_{j}\right)$ would create a center of $\Gamma_{j}$, hence of $\Gamma$, in the same way as above. This observation now implies that the leaves of our two foliations are orthogonal to each other in the sense of inducing the desired product structure for the metric on $\tilde{M}$.

Theorem 3. Let $\Gamma$ be a centerfree group of isometries of a product Riemannian manifold $X=X_{1} \times \ldots X_{k}(k \geq 2)$, acting freely and properly discontinuously with compact quotient $X / \Gamma$. Assume that $\Gamma$ acts irreducibly in the sense that no finite index subgroup of $\Gamma$ splits as a product. Assume that $M$ is a compact Riemannian manifold of nonpositive sectional curvature homotopically equivalent to $X / \Gamma$. Then there exists a totally geodesic diffeomorphism

$$
f: X / \Gamma \rightarrow M
$$

and $\tilde{M}$ and $X$ are Riemannian homogeneous.

Proof. We claim that the projection $\Gamma_{j}$ of $\Gamma$ into the isometry group of $X_{j}$, $I\left(X_{j}\right)$, acts densely on $X_{j}, j=1, \ldots k$. Assume on the contrary that it acts properly discontinuously on some maximal subset $\Omega_{j}$ of $X_{j}$. We conclude that $\Omega_{j}=X_{j}$; namely, any $p \in X_{j} \backslash \Omega_{j}$ by the maximal choice of $\Omega_{j}$ has the property that there exists a nontrivial sequence $\left(\gamma_{\nu}\right)_{\nu \in \mathbb{N}} \subset \Gamma_{j}$ for which

$$
\gamma_{\nu} p \rightarrow p_{0}
$$

for some $p_{0} \in X_{j}$. Take any $q \in \Omega_{j}$. Then, since $\Gamma_{j}$ acts by isometries,

$$
d\left(\gamma_{\nu} p, \gamma_{\nu} q\right)=d(p, q)
$$


Hence, $\gamma_{\nu} q$ remains bounded, hence converges after selection of a subsequence, contradicting the definition of $\Omega_{j}$. Thus, indeed, $\Omega_{j}=X_{j}$, and $\Gamma_{j}$ acts properly discontinuously on $X_{j}$.

We shall now conclude that $\Gamma$ is reducible. Let $X_{j}^{\prime}$ be the product of the factors of $X$ different from $X_{j}$. We claim that the sets $X_{j}^{\prime} \times\{p\}$ for $p \in X_{j}$ yield a foliation of $X / \Gamma$ with compact leaves. Namely, any point in such a set $X_{j}^{\prime} \times\{p\}$ is identified only with a discrete set of points in other such sets, because $\Gamma_{j}$ acts properly discontinuously on $X_{j}$. In particular, in any fundamental region for the action of $\Gamma$, only finitely many such sets are equivalent under the action of $\Gamma$, where we call two sets equivalent if they contain points identified by the action of $\Gamma$. This easily implies compactness of the leaves.

Let $\Gamma_{j}^{\prime}$ be the projection of $\Gamma$ onto the isometry group $I\left(X_{j}^{\prime}\right)$. Since $\Gamma$ has compact quotient $X / \Gamma$, and since the sets $X_{j}^{\prime} \times\{p\}$ yield compact leaves in $X / \Gamma, \Gamma_{j}^{\prime n}:=\left\{\gamma \in \Gamma_{j}^{\prime}:(e, \gamma) \in \Gamma\right\}$ has to act with compact quotient on each such set. Since $\Gamma_{j}$ and $\Gamma_{j}^{\prime}$ commute, each $\gamma \in \Gamma_{j}$ yields an automorphism of any such set $\left(X_{j}^{\prime} \times\{p\}\right) / \Gamma_{j}^{\prime n}$.

As in the proof of Thm. 2, we look at the family of harmonic maps

$$
f_{p}:\left(X_{j}^{\prime} \times\{p\}\right) / \Gamma_{j}^{\prime n} \rightarrow M
$$

induced by the homotopy equivalence between $X / \Gamma$ and $M$. Again, these maps form a parallel family $f$ that is continuous in $p$. Since $\gamma \in \Gamma_{j}$ yields an automorphism of $\left(X_{j}^{\prime} \times\{p\}\right) / \Gamma_{j}^{\prime n},\left.f\right|_{\left(X_{j}^{\prime} \times\{p\}\right) / \Gamma_{j}^{\prime n}}$ and $\left.\gamma \circ f\right|_{\left(X_{j}^{\prime} \times\{p\}\right) / \Gamma_{j}^{\prime n}}$ induce homotopic harmonic maps from compact spaces, and by uniqueness of harmonic maps (see $\S 1$ ) again, they can be joined by a parallel family. Noting that $\Gamma_{j}^{\prime n}$ is a normal subgroup of $\Gamma$, we therefore get a flat bundle with fiber $f\left(\left(X_{j}^{\prime} \times\{p\}\right) / \Gamma_{j}^{\prime n}\right)$ over the base $f\left(X_{j} / \Gamma_{j}\right)$. Thus, $\gamma \in \Gamma_{j}$ induces an automorphism of the fiber. By the Arzela-Ascoli theorem, these automorphisms can only be contained in finitely many homotopy classes of selfmaps of the fiber. On the other hand, any nontrivial such automorphism homotopic to the identity generates a nontrivial center of $\Gamma_{j}^{\prime n}$, hence also of $\Gamma$, as in the proof of Thm. 2. Thus, $\Gamma_{j}$ induces only finitely many automorphisms of the fiber. Therefore, a finite cover of this flat bundle splits as a global product. Since we have a homotopy equivalence between $X / \Gamma$ and $M$, i.e. a map between $X$ and $\tilde{M}$ equivariant w.r.t. the $\Gamma$-actions on these spaces, we may pull this product back to $X$. Now $\Gamma$ and $\Gamma_{j} \times \Gamma_{j}^{\prime n}$ are commensurable, since they both are subgroups of $\Gamma_{j} \times \Gamma_{j}^{\prime}$ with a quo- 
tient of finite nonzero measure. Therefore, we obtain a contradiction to the assumption that $\Gamma$ acts irreducibly on $X$.

In order to show density of the action, however, it remains to exclude the intermediate case where $\Gamma_{j}$ has a nondiscrete orbit whose closure in $X_{j}$, however, is not all of $X_{j}$. In that case, we have a family of leaves $\bar{\Sigma}(t)$ in $X_{j}$, depending on some parameter $t$, obtained as the orbits of the closure $\bar{\Gamma}_{j}$ of $\Gamma_{j}$ in the isometry group $I\left(X_{j}\right)$. Since we have more than one leaf, $\bar{\Gamma}_{j}$ has to contain more than one connected component. The projections $\Sigma(t)$ of $\bar{\Sigma}(t)$ form a foliation of $X / \Gamma$ with compact leaves. For every leaf, we let $f_{t}: \Sigma(t) \rightarrow M$ be the harmonic map in the homotopy class of $f_{\left.\right|_{\Sigma(t)}}$. Let $\gamma \in \bar{\Gamma}_{j}$ be contained in a connected component of $\bar{\Gamma}_{j}$ not containing the identity. $\gamma$ then does not map $\bar{\Sigma}(t)$ onto itself, but onto another leaf $\bar{\Sigma}\left(t^{\prime}\right)$. It therefore normalizes the subgroup of $\bar{\Gamma}_{j}$ of those elements mapping $\bar{\Sigma}(t)$ onto itself, at least for generic $t$. Namely, the quotient of $\bar{\Gamma}_{j}$ by the identity component acts properly discontinuously on the leaf space $\{t\}$, and therefore, the set of its fixed points is discrete. For $t$ not in this fixed point set, only elements of $\bar{\Gamma}_{j}$ contained in the identity component map $\bar{\Sigma}(t)$ onto itself, and $\gamma$ normalizes this subgroup.

We then obtain a nontrivial homotopy between the two harmonic maps $f_{t}$ and $\gamma \circ f_{t}$. We then obtain a vector field along $f_{t}(\Sigma(t))$, and performing this construction for every $t$, along $M$ that allows us to create a center in the same manner as in the argument for the case where $\Gamma_{j}$ was assumed to act properly discontinuously. Since we assume, however, that $\Gamma$ is center free, we conclude that any orbit of $\Gamma_{j}$ is dense in $X$. Therefore, $\bar{\Gamma}_{j}$ acts transitively on $X_{j}$, and $X_{j}$ is a homogeneous space.

The proof of Thm. 3 is now completed by the following

Lemma 1. Let the discrete group $\Gamma$ operate properly discontinuously and isometrically on the Riemannian manifold $X=X_{1} \times \ldots X_{k}(k \geq 2)$ with compact quotient $X / \Gamma$. Let $\rho: \Gamma \rightarrow I(Y)$ be a homomorphism into the isometry group of the Riemannian manifold $Y$ of nonpositive sectional curvature, and let $f: X \rightarrow Y$ be a $\rho$-equivariant harmonic map. Then the restriction of $f$ to each factor of $X$ is harmonic itself, and if $V$ and $W$ are tangent to different factors through $p \in X$, then

$$
\nabla^{2} f(p)(V, W)=0
$$

If $\Gamma$ has no center and operates irreducibly, then $f$ is totally geodesic. 
Proof. For simplicity of notation, we assume $k=2$, i.e.

$$
X=X_{1} \times X_{2} \text {. }
$$

The general case can be reduced to that one by an easy induction. We let

$$
\left(e_{\alpha}\right)_{\alpha=1, \ldots n}
$$

be an orthonormal frame at $p \in X$, with $e_{1}, \ldots e_{m_{1}}$ tangent to $X_{1}$, and $e_{m_{1}+1}, \ldots e_{m_{1}+m_{2}}$ tangent to $X_{2}$. We let indices $\alpha_{1}, \beta_{1}, \ldots$ range from 1 to $m_{1}, \alpha_{2}, \beta_{2}, \ldots$ from $m_{1}+1$ to $m_{1}+m_{2}, \alpha, \beta, \ldots$ from 1 to $m=m_{1}+m_{2}$. The standard Bochner formula for harmonic maps gives with the usual summation convention and the Riemannian metric $\langle\cdot, \cdot\rangle$ of $Y$, the Ricci tensor $\operatorname{Ric}^{X}$ of $X$ and the curvature $R^{Y}$ of $Y$

$$
\begin{aligned}
\int_{X / \Gamma}\left\langle f_{\alpha \alpha}, f_{\beta \beta}\right\rangle= & \int_{X / \Gamma}\left\langle f_{\alpha \beta}, f_{\alpha \beta}\right\rangle+\int_{X / \Gamma}\left\langle R i c^{X}\left(f_{\alpha}\right), f_{\alpha}\right\rangle \\
& -\int_{X / \Gamma}\left\langle R^{Y}\left(f_{\alpha}, f_{\beta}\right) f_{\beta}, f_{\alpha}\right\rangle
\end{aligned}
$$

(1) just follows from integrating by parts and the definition of the curvature tensors. In the same way, we obtain for $j=1,2$

$$
\begin{aligned}
\int_{X / \Gamma}\left\langle f_{\alpha_{j} \alpha_{j}}, f_{\beta_{j} \beta_{j}}\right\rangle= & \int_{X / \Gamma}\left\langle f_{\alpha_{j} \beta_{j}}, f_{\alpha_{j} \beta_{j}}\right\rangle+\int_{X / \Gamma}\left\langle R i c^{X}\left(f_{\alpha_{j}}\right), f_{\alpha_{j}}\right\rangle \\
& -\int_{X / \Gamma}\left\langle R^{Y}\left(f_{\alpha_{j}}, f_{\beta_{j}}\right) f_{\beta_{j}}, f_{\alpha_{j}}\right\rangle
\end{aligned}
$$

Since $f$ is harmonic, $f_{\alpha \alpha}=0$, and so the left hand side of (1) vanishes. Noting also that

$$
\left\langle\operatorname{Ric}^{X} f_{\alpha_{1}}, f_{\alpha_{2}}\right\rangle=0
$$

for all $\alpha_{1}, \alpha_{2}$, since $X=X_{1} \times X_{2}$ is metrically a product, we subtract (2.1) and(2.2) from (1) to obtain

$$
\begin{aligned}
0= & \int_{X / \Gamma}\left\langle f_{\alpha_{1} \alpha_{2}}, f_{\alpha_{1} \alpha_{2}}\right\rangle+\int_{X / \Gamma}\left\langle f_{\alpha_{1} \alpha_{1}}, f_{\beta_{1} \beta_{1}}\right\rangle+\int_{X / \Gamma}\left\langle f_{\alpha_{2} \alpha_{2}}, f_{\beta_{2} \beta_{2}}\right\rangle \\
& -\int_{X / \Gamma}\left\langle R^{Y}\left(f_{\alpha_{1}}, f_{\alpha_{2}}\right) f_{\alpha_{2}}, f_{\alpha_{1}}\right\rangle .
\end{aligned}
$$

Since $Y$ has nonpositive sectional curvature and the other integrands in (3) are (sums of) squares, all integrands in (3) have to vanish identically. In particular

$$
f_{\alpha_{1} \alpha_{1}}=0=f_{\alpha_{2} \alpha_{2}}
$$


so that the restriction of $f$ to each factor is harmonic, and

$$
f_{\alpha_{1} \alpha_{2}}=0 \text { for all } \alpha_{1}, \alpha_{2},
$$

so that the Hessian of $f$ vanishes in mixed directions. In case $\Gamma$ is centerfree and acts irreducibly, we shall now show

$$
f_{\alpha_{1} \beta_{1}}=0 \text { for all } \alpha_{1}, \beta_{1} \text {. }
$$

By the same argument then also $f_{\alpha_{2} \beta_{2}}=0$ for all $\alpha_{2}, \beta_{2}$, and altogether the Hessian of $f$ vanishes and $f$ is totally geodesic. (6), however, easily follows from the fact that

$$
\left\langle f_{\alpha_{1}}, f_{\beta_{1}}\right\rangle
$$

is invariant not only under $\Gamma_{2}$ (because of (5)) but also under $\Gamma_{1}\left(\Gamma_{j}\right.$ is the projection of $\Gamma$ onto the isometry group of $X_{j}$ ) and that the latter group acts densely in $X_{1}$ by what has been shown in the preceding arguments in the proof of Thm. 3. This completes the proof of the Lemma and of Thm. 3 .

\section{References.}

[A1] S.I. Al'ber, On n-dimensional problems in the calculus of variations in the large, Sov. Math. Dokl. 5 (1964), 700-704.

[A2] S.I. Al'ber, Spaces of mappings into a manifold with negative curvature, Sov. Math. Dokl. 9 (1967), 6-9.

[BGS] W. Ballmann, M. Gromov, and V. Schroeder, Manifolds of nonpositive curvature, Birkhäuser, 1985.

[B] A. Borel, Density properties for certain subgroups of semi-simple groups without compact components, Ann. Math. 72 (1960), 179-188.

[E] P. Eberlein, Rigidity of lattices of nonpositive curvature, Ergodic Theory and Dyn. Systems, 3 (1983), 47-85.

[ES] J. Eells and J. Sampson, Harmonic mappings of Riemannian manifolds, Am. J. Math. 85 (1964), 109-160.

[GW] D. Gromoll and J. Wolf, Some relations between the metric structure and the algebraic structure of the fundamental group in manifolds of nonpositive curvature, BAMS 77 (1971), 545-552.

[GS] M. Gromov and R. Schoen, Harmonic maps into singular spaces and padic superrigidity for lattices in groups of rank one, Publ. Math. IHES, 76 (1992), 165-246. 
[H] P. Hartman, On homotopic harmonic maps, Can. J. Math. 19 (1967), 673687.

[J1] J. Jost, Nonlinear methods in Riemannian and Kählerian geometry, Birkhäuser, DMV Seminar Vol. 10, $2^{\text {nd }}$ edition, 1991.

[J2] J. Jost, Equilibrium maps between metric spaces, Calc. Var. 2 (1994), 173204.

[J3] J. Jost, Nonpositive curvature: geometric and analytic aspects, Birkhäuser, 1997.

[J4] J. Jost, Generalized Dirichlet forms and harmonic maps, Calc. Var. 5 (1997), 1-19.

[JY] J. Jost and S. T. Yau, Harmonic maps and superrigidity, Proc. Sym. Pure Math. 54, Part I, (1993), 245-280.

[LY] B. Lawson and S. T. Yau, Compact manifolds of nonpositive curvature, J. Diff. Geom. 7 (1972), 211-228.

[MSY] N. Mok, Y.-T. Siu, and S. K. Yeung, Geometric superrigidity, Inv. math. 113 (1993), 57-84.

[N] I. Nikolaev, Synthetic methods in Riemannian geometry, Lecture Notes.

[SY] R. Schoen and S. T. Yau, Compact group actions and the topology of manifolds with non-positive curvature, Top. 18 (1979), 361-380.

[W] G. Whitehead, Elements of homotopy theory, Springer, GTM 61, 1978.

ReCeived May 12, 1994 AND REVised June 22, 1998. 
JÜRGEN JOST

MAX-PlanCK-Institute for Mathematics

IN THE SCIENCES

INSELSTR. 22-26

D-04103, LEIPZIG

Germany

E-MAIL: JOST@MIS.MPG.DE

AND

S.-T. YAU

Department of Mathematics

1 OXFORD ST.

HARVARD UNIVERSITY

CAMBridge, MA 02138

U.S.A.

E-MAIL:YAU@MATH.HARVARD.EDU 